\title{
Predictions for multiplicities and flow harmonics in 5.44 TeV Xe+Xe collisions at the CERN Large Hadron Collider
}

\author{
K. J. Eskola, ${ }^{1,2}$ H. Niemi, ${ }^{1,2,3}$ R. Paatelainen, ${ }^{2,4}$ and K. Tuominen ${ }^{2,4}$ \\ ${ }^{1}$ University of Jyvaskyla, Department of Physics, P.O. Box 35, FI-40014 University of Jyvaskyla, Finland \\ ${ }^{2}$ Helsinki Institute of Physics, P.O. Box 64, FI-00014 University of Helsinki, Finland \\ ${ }^{3}$ Institut für Theoretische Physik, Johann Wolfgang Goethe-Universität, Max-von-Laue-Str. 1, D-60438 Frankfurt am Main, Germany \\ ${ }^{4}$ Department of Physics, P.O. Box 64, FI-00014 University of Helsinki, Finland
}

(Received 29 November 2017; published 19 March 2018)

\begin{abstract}
We present the event-by-event next-to-leading-order perturbative-QCD + saturation + viscous hydrodynamics (EKRT) model predictions for the centrality dependence of the charged hadron multiplicity in the pseudorapidity interval $|\eta| \leqslant 0.5$, and for the centrality dependence of the charged hadron flow harmonics $v_{n}\{2\}$ obtained from two-particle cumulants, in $\sqrt{s_{N N}}=5.44 \mathrm{TeV} \mathrm{Xe}+\mathrm{Xe}$ collisions at the CERN Large Hadron Collider. Our prediction for the 0-5\% central charged multiplicity is $d N_{\mathrm{ch}} / d \eta=1218 \pm 46$. We also predict $v_{n}\{2\}$ in $\mathrm{Xe}+\mathrm{Xe}$ collisions to increase more slowly from central towards peripheral collisions than those in a $\mathrm{Pb}+\mathrm{Pb}$ system. We find that at $10, \ldots, 50 \%$ centralities $v_{2}\{2\}$ is smaller and $v_{3}\{2\}$ is larger than in the $\mathrm{Pb}+\mathrm{Pb}$ system while $v_{4}\{2\}$ is of the same magnitude in both systems. We also find that the ratio of flow harmonics in $\mathrm{Xe}+\mathrm{Xe}$ collisions and in $\mathrm{Pb}+\mathrm{Pb}$ collisions shows a slight sensitivity to the temperature dependence of the shear-viscosity-to-entropy ratio. As we discuss here, the new nuclear mass-number systematics especially in the flow harmonics serves as a welcome further constraint for describing the space-time evolution of a heavy-ion system and for determining the shear viscosity and other transport properties of strongly interacting matter.
\end{abstract}

DOI: 10.1103/PhysRevC.97.034911

\section{INTRODUCTION}

Ultrarelativistic heavy-ion collisions at the CERN Large Hadron Collider (LHC) and BNL Relativistic Heavy Ion Collider (RHIC) probe the collectivity in quantum chromodynamics (QCD) by producing strongly interacting QCD matter at high temperatures and vanishing net-baryon number densities. To correctly interpret the measurements, it is of pivotal importance to understand the primary production dynamics of the quark-gluon plasma (QGP) and know the transport properties of the produced QCD matter. For this, on the one hand, one needs QCD-based predictive modeling to describe the production of the system at various collision energies and nuclei [1-11]. Combining this with a fluid-dynamical spacetime evolution, event by event, then enables the computation of a multitude of small transverse-momentum $\left(p_{T}\right)$ finalstate observables [12-23]. On the other hand, for extracting the uncertainties of the QCD matter properties and other theory-input parameters from the measurements, one needs a statistical multiobservable (global) analysis [24-29].

The Bayesian global analysis of small- $p_{T}$ observables in the $\mathrm{LHC} \mathrm{Pb}+\mathrm{Pb}$ collisions, discussed in Refs. [26-28], quite

Published by the American Physical Society under the terms of the Creative Commons Attribution 4.0 International license. Further distribution of this work must maintain attribution to the author(s) and the published article's title, journal citation, and DOI. Funded by $S C O A P^{3}$. strongly suggests that the initial-state dynamics in heavy-ion collisions is correctly captured by the saturation models nextto-leading-order (NLO) event-by-event (EbyE) EKRT [18 $]^{1}$ and IP-Glasma [14]. Interestingly, even though these models approach the saturation from different limits-EKRT from collinear factorization and perturbative QCD (pQCD), and IP-Glasma from classical gluon fields and small- $x$ QCDit is the same phenomenon, dominance of gluon fusions (nonlinearities) at small transverse momenta, that regulates and controls the produced initial multiplicities in both frameworks.

The NLO EbyE EKRT model, where the computed QCDmatter initial states are combined with shear-viscous fluid dynamics, predicts successfully the centrality- and cms-energy dependencies of hadron multiplicities and their $p_{T}$ distributions, flow coefficients, relative elliptic flow fluctuations, and various flow correlators in 2.76 and $5.02 \mathrm{TeV} \mathrm{Pb}+\mathrm{Pb}$ collisions at the LHC and $200 \mathrm{GeV} \mathrm{Au}+\mathrm{Au}$ collisions at RHIC [18,30]. For a recent review, see Ref. [31]. Importantly, the simultaneous EKRT analysis of all these observables suggests systematically that the temperature dependence of the shearviscosity-to-entropy $(\eta / s)$ ratio of the produced QCD matter is in the range shown by Fig. 1. As expected, similar results for the range of $\eta / s(T)$ were then obtained also in the Bayesian analysis - see Ref. [27] for a recent review.

What has been missing so far, however, is the nuclear massnumber $(A)$ systematics at the LHC. The short ${ }^{129} \mathrm{Xe}+{ }^{129} \mathrm{Xe}$

${ }^{1}$ Named after the authors of Ref. [3]. 


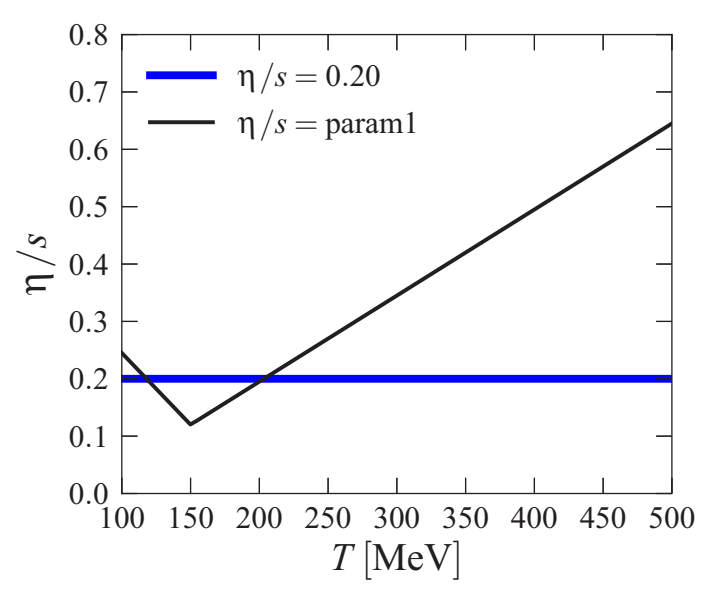

FIG. 1. The two parametrizations of the temperature dependence of the shear-viscosity-to-entropy ratio which in the NLO EbyE EKRT model $[18,30]$ give the best overall fit to the various small- $p_{T}$ LHC and RHIC observables.

run with nucleon-nucleon cms energy $\sqrt{s_{N N}}=5.44 \mathrm{TeV}$ at the LHC in October 2017 now fills this gap conveniently, offering important further observables to be included in the global analyses. Motivated by this LHC run, in this article we will present our NLO EbyE EKRT model predictions for the centrality dependencies of the charged hadron multiplicities in the pseudorapidity interval $|\eta| \leqslant 0.5$ and of the flow coefficients $v_{n}\{2\}$ computed from the two-particle cumulants. These predictions are obtained by using the two $\eta / s(T)$ parametrizations of Fig 1 . In addition to the $A$ systematics, it will be interesting to see whether the $v_{n}\{2\}$ in the $\mathrm{Xe}+\mathrm{Xe}$ collisions would show any increased sensitivity to $\eta / s(T)$ relative to the larger $\mathrm{Pb}+\mathrm{Pb}$ system.

Below, we will first outline the procedure for computing these predictions, then show and discuss the obtained results. A detailed account of the model details can be found in Ref. [18].

\section{KEY STEPS FOR XE+XE PREDICTIONS}

The first step is to compute the transverse-area density of minijet transverse energy, $d E_{T} / d^{2} \mathbf{r}$, in a nuclear collision at a given cms energy $\sqrt{s_{N N}}$ and impact parameter $\mathbf{b}$, accounting for minijets above a transverse momentum cut-off $p_{0} \gg \Lambda_{\mathrm{QCD}}$ in a central rapidity unit $\Delta y$,

$$
\frac{d E_{T}}{d^{2} \mathbf{r}}=T_{A}\left(\mathbf{r}_{1}\right) T_{A}\left(\mathbf{r}_{2}\right) \sigma\left\langle E_{T}\right\rangle_{p_{0}, \Delta y, \beta} .
$$

The nuclear thickness functions $T_{A}$ give the collision geometry, with $\mathbf{r}_{1,2}=\mathbf{r} \mp \mathbf{b} / 2$, where $\mathbf{r}$ is the transverse coordinate. For Xe (isotope $A=129$ ) we compute the $T_{A}$ using the WoodsSaxon nuclear density with standard radius parameters $R_{A}=$ $1.12 A^{1 / 3}-0.86 A^{-1 / 3}$ and $d=0.54 \mathrm{fm}$. The $E_{T}$-weighted minijet cross section $\sigma\left\langle E_{T}\right\rangle_{p_{0}, \Delta y, \beta}[1]$ is computed in collinear factorization and NLO pQCD using the subtraction method [32-34]. This involves the squared invariant amplitudes for $2 \rightarrow 2$ and $2 \rightarrow 3$ parton scatterings [35,36], CTEQ6M parton distribution functions [37] supplemented with EPS09s transverse-coordinate dependent nuclear effects [38]. Also included in $\sigma\left\langle E_{T}\right\rangle$ are the infrared(IR)- and collinear(CL)-safe

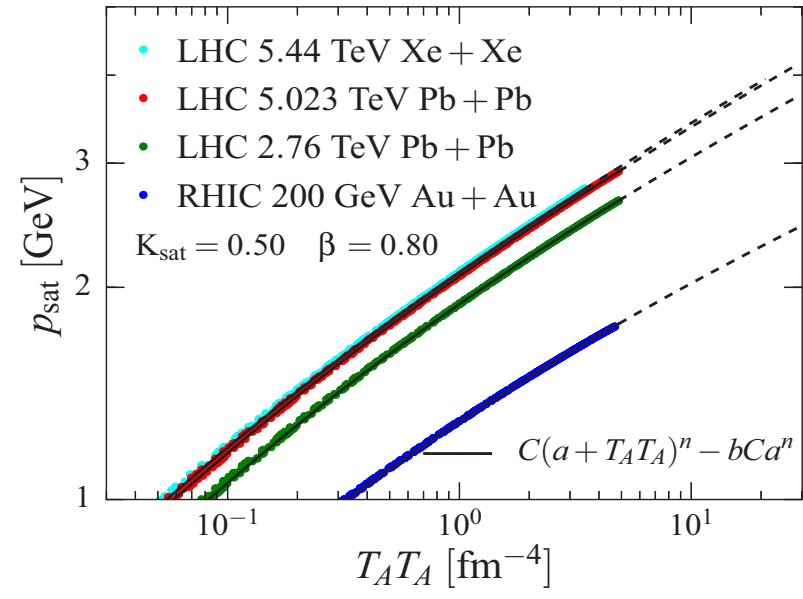

FIG. 2. Saturation momenta vs. the thickness function product, computed in $5.44 \mathrm{TeV} \mathrm{Xe}+\mathrm{Xe}$ collisions for $|\mathbf{b}|=0,6.59$, and $8.27 \mathrm{fm}$, with $\beta=0.8$ and $K_{\text {sat }}=0.5$ corresponding to $\eta / s(T)$ of param1. The previous results $[18,30]$ for 5.023 and $2.76 \mathrm{TeV} \mathrm{Pb}+\mathrm{Pb}$ collisions at the LHC, and $200 \mathrm{GeV} \mathrm{Au+Au}$ collisions at RHIC are shown for comparison. The parametrization $p_{\mathrm{sat}}\left(T_{A} T_{A} ; K_{\mathrm{sat}}, \beta\right)$ of the $\mathrm{Xe}+\mathrm{Xe}$ result is from Table I, and those for the $\mathrm{Pb}+\mathrm{Pb}$ and $\mathrm{Au}+\mathrm{Au}$ cases are from Refs. $[18,30]$. The order of the results from top to bottom is the same as in the legend. The QCD calculations are presented by points and the parametrizations by solid and dashed lines.

measurement functions which define the minijet $E_{T}$ as the scalar sum of minijet transverse momenta in $\Delta y$, as well as IR/CL-safe definitions of the cut-off $p_{0}$ and of the minimum $E_{T}\left(E_{T} \geqslant \beta p_{0} ; 0 \leqslant \beta \leqslant 1\right)$ allowed in $\Delta y[10,33,34]$. For a detailed formulation of $\sigma\left\langle E_{T}\right\rangle$, see Refs. [10,18]. In the first step, we thus compute the $\sigma\left\langle E_{T}\right\rangle$ various times: for an array of $\beta$, for a range of $p_{0}$ values around the expected saturation momentum, for various values of $\mathbf{b}$ and for a lattice of $\mathbf{r}$ in the first quadrant of the transverse plane.

The second step is then to find the mapping between the collision geometry given by $T_{A}\left(\mathbf{r}_{1}\right) T_{A}\left(\mathbf{r}_{2}\right)$ and the saturation scale $p_{0}=p_{\text {sat }}\left(\sqrt{s_{N N}}, A, \mathbf{r}, \mathbf{b} ; \beta, K_{\text {sat }}\right)$ which dictates gluon production locally at each $\mathbf{r}$. The saturation momenta are obtained as the solutions of the following saturation criterion $[10,11]$ which derives from the limit where the minijet $E_{T}$ production starts to be dominated by higher-order fusion processes $(n \geqslant 2) \rightarrow 2$ over the usual $2 \rightarrow 2$ ones:

$$
\frac{\mathrm{d} E_{T}}{\mathrm{~d}^{2} \mathbf{r}}\left(p_{0}, \sqrt{s_{N N}}, A, \Delta y, \mathbf{r}, \mathbf{b}, \beta\right)=\frac{K_{\text {sat }}}{\pi} p_{0}^{3} \Delta y .
$$

Here, $K_{\text {sat }}$ is a proportionality constant whose value we now know from Ref. [18] where it was obtained by normalizing the computed charged-particle multiplicity $d N_{\mathrm{ch}} / d \eta$ to the one measured by ALICE in $|\eta| \leqslant 0.5$ in $0-5 \%$ central $2.76 \mathrm{TeV}$ $\mathrm{Pb}+\mathrm{Pb}$ collisions. Figure 2 shows the values of $p_{\text {sat }}$ obtained for the current LHC 5.44 TeV Xe+Xe case for three different values of $|\mathbf{b}|$ with $K_{\text {sat }}=0.5$ and $\beta=0.8$ that correspond to our $\eta / s=$ param 1 parametrization. Our earlier results for the LHC 5.023 TeV and 2.76 TeV Pb+Pb collisions and RHIC $200 \mathrm{GeV} \mathrm{Au}+\mathrm{Au}$ collisions from Refs. [18,30] are shown for comparison. As expected [18,39], $p_{\text {sat }}$ in the $\mathrm{Xe}+\mathrm{Xe}$ system 
TABLE I. Parametrization $p_{\text {sat }}\left(T_{A} T_{A} ; K_{\text {sat }}, \beta\right)=C\left[a+\rho_{A A}\right]^{n}-$ $b C a^{n}$ in $5.44 \mathrm{TeV} \mathrm{Xe}+\mathrm{Xe}$ collisions for $K_{\text {sat }} \in[0.4,2.0]$ and $\beta<0.9$. The $\left(K_{\text {sat }}, \beta\right)$ dependence of $a, b, C$ and $n$ (see below) is parametrized as $P_{i}\left(K_{\mathrm{sat}}, \beta\right)=a_{i 0}+a_{i 1} K_{\mathrm{sat}}+a_{i 2} \beta+a_{i 3} K_{\mathrm{sat}} \beta+a_{i 4} \beta^{2}+a_{i 5} K_{\mathrm{sat}}^{2}$.

\begin{tabular}{lrrrr}
\hline \hline$P_{i} \rightarrow$ & \multicolumn{1}{c}{$C$} & \multicolumn{1}{c}{$n$} & \multicolumn{1}{c}{$a$} & \multicolumn{1}{c}{$b$} \\
\hline$a_{i 0}$ & 3.9593855 & 0.1454605 & -0.0034895 & 0.8309836 \\
$a_{i 1}$ & -0.7184810 & -0.0176172 & 0.0162876 & -0.0592489 \\
$a_{i 2}$ & 0.6052426 & -0.0244353 & -0.0004785 & 0.0869927 \\
$a_{i 3}$ & 0.0748967 & -0.0030068 & 0.0074064 & -0.0000669 \\
$a_{i 4}$ & -1.5647924 & 0.0546230 & -0.0023656 & -0.2067018 \\
$a_{i 5}$ & 0.1347936 & 0.0057852 & -0.0026187 & 0.0234235 \\
\hline \hline
\end{tabular}

depends again only on the product $T_{A}\left(\mathbf{r}_{1}\right) T_{A}\left(\mathbf{r}_{2}\right)$ and not on $\mathbf{r}$ and $\mathbf{b}$ separately, i.e., $p_{\text {sat }}(\mathbf{r}, \mathbf{b}) \approx p_{\text {sat }}\left(T_{A}\left(\mathbf{r}_{1}\right) T_{A}\left(\mathbf{r}_{2}\right)\right)$. This is the key feature making our EbyE framework possible in practice.

For handling the densest nuclear overlap regions in the fluctuating EbyE case (see Fig. 2, dashed lines), we must still parametrize the $T_{A} T_{A}$ dependence of $p_{\text {sat }}$. For enabling further studies of the uncertainties related to the fixing of $K_{\text {sat }}$ and $\beta$, we also parametrize the $K_{\text {sat }}$ and $\beta$ dependence of $p_{\text {sat }}$ similarly to what we did before $[18,30]$. The outcome is shown in Table I and Fig. 2.

The third step is to prepare the initial states for fluid dynamics event by event. As in Refs. [18,30], we obtain the EbyE fluctuating initial energy densities by setting a Gaussian gluon transverse density distribution of a width $\sigma=0.43 \mathrm{fm}$ around each nucleon sampled from the Woods-Saxon density distribution. The thickness functions $T_{A}$ are then computed by locally summing up the gluon transverse densities. For fixed $K_{\text {sat }}, \beta$, the obtained product $T_{A} T_{A}$ now maps to $p_{\text {sat }}$ according to Fig. 2 and Table I, and we can compute the local energy density at the local formation time $\tau_{s}(\mathbf{r})=1 / p_{\text {sat }}(\mathbf{r})$ as

$$
\varepsilon\left(\mathbf{r}, \tau_{s}(\mathbf{r})\right)=\frac{d E_{T}\left(p_{\text {sat }}\right)}{d^{2} \mathbf{r}} \frac{1}{\tau_{s}(\mathbf{r}) \Delta y}=\frac{K_{\text {sat }}}{\pi}\left[p_{\text {sat }}(\mathbf{r})\right]^{4} .
$$

We assume this to be valid for $p_{\text {sat }} \geqslant p_{\text {sat }}^{\min }=1 \mathrm{GeV}$. To start the fluid-dynamic simulation at a constant proper time $\tau_{0}=$ $1 / p_{\mathrm{sat}}^{\min }=0.2 \mathrm{fm}$, we evolve these energy densities locally from $\tau_{s}(\mathbf{r})$ to $0.2 \mathrm{fm}$ with $1 \mathrm{D}$ Bjorken hydrodynamics. Finally, we treat the dilute edges of the system as explained in [18] [Eq. (34) there $\left.{ }^{2}\right]$.

The fourth step is the fluid-dynamic runs, event by event. Our setup is identical to that of Refs. [18,30], i.e., we apply second-order dissipative relativistic $2+1$ dimensional hydro with transient fluid-dynamics equation of motion for the shearstress tensor as in Refs. [42,43]. Our QCD-matter equation of state is $s 95 p$-PCE-v1 [44] with chemical decoupling taking place at $T_{\text {chem }}=175 \mathrm{MeV}$, and kinetic freeze-out at $T_{\mathrm{dec}}=$ $100 \mathrm{MeV}$. Initially the shear-stress tensor $\pi^{\mu \nu}$ and transverse flow are assumed to be zero, and on the freeze-out surface the viscous $\delta f$ corrections are $\propto p_{\mu} p_{\nu} \pi^{\mu \nu}$. We do not consider the bulk viscosity or heat conductivity but account for the shear viscosity and its temperature dependence as shown in Fig. 1.

\footnotetext{
${ }^{2}$ Now $\sigma_{N N}=70.53 \mathrm{mb}$, obtained from the parametrization in Refs. [40,41].
}

\section{RESULTS}

Figure 3 shows our prediction for the centrality dependence of the charged particle multiplicity in $5.44 \mathrm{TeV} \mathrm{Xe}+\mathrm{Xe}$ collisions, computed for the $\eta / s$ parametrizations of Fig. 1. Our previous results for $2.76 \mathrm{TeV} \mathrm{Pb}+\mathrm{Pb}$ collisions and 200 $\mathrm{GeV} \mathrm{Au}+\mathrm{Au}$ collisions [18], and predictions for $5.023 \mathrm{TeV}$ $\mathrm{Pb}+\mathrm{Pb}$ collisions [30], as well as the ALICE data [45,46] for 2.76 and $5.02 \mathrm{TeV}$, and the STAR [47] and PHENIX [48] data for the $200 \mathrm{GeV}$ collisions, are shown for comparison.

We emphasize that the centralmost ALICE datapoint at 2.76 $\mathrm{TeV}$ - and this one point only-has been used for fixing the normalization of our results $\left(K_{\text {sat }}=0.63(0.5)\right.$ for $\eta / s=0.2$ (param1) from [18]). Thus, the error bar of this ALICE point translates into a normalization uncertainty in our $5.44 \mathrm{TeV}$ prediction as shown by the yellow error band. The normalization was done iteratively to a few percent accuracy, causing the corresponding difference between the predicted centralmost 5.44 TeV multiplicities (dashed curves). We refrain here from further fine-tuning, because the relative normalization uncertainties in our centralmost $2.76 \mathrm{TeV}$ multiplicity are transmitted practically directly into those of our $5.44 \mathrm{TeV}$ multiplicity prediction. We account for these normalization uncertainties as follows, using the centralmost multiplicities given in the caption of Fig. 3: Multiplying the param1 result 1199 by $1601 / 1576$ gives 1218 , and estimating the error as $(60 / 1601) \times 1218=46$, we arrive at our best prediction for the charged multiplicity in $0-5 \%$ central $5.44 \mathrm{TeV} \mathrm{Xe}+\mathrm{Xe}$ collisions:

$$
\left.\frac{d N_{\mathrm{ch}}}{d \eta}\right|_{|\eta| \leqslant 0.5} ^{\mathrm{Xe}+\mathrm{Xe}}=1218 \pm 46 .
$$

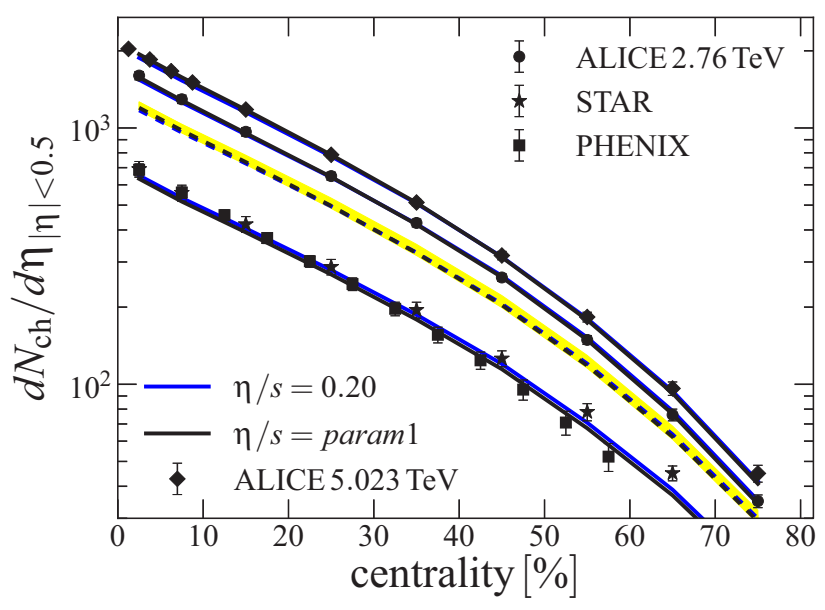

FIG. 3. The NLO EbyE EKRT model prediction for the centrality dependence of charged hadron multiplicity in $5.44 \mathrm{TeV} \mathrm{Xe}+\mathrm{Xe}$ collisions at the LHC, computed for the two $\eta / s(T)$ parametrizations of Fig. 1. The results of Refs. [18,30], the $\mathrm{Pb}+\mathrm{Pb}$ measurements by ALICE at 2.76 and 5.02 TeV [45,46], and the Au+Au measurements at $200 \mathrm{GeV}$ by STAR [47] and PHENIX [48] are also shown. The computed centralmost multiplicities for 5.44 (2.76) $\mathrm{TeV}$ are 1172 (1542) for $\eta / s=0.2$, and 1199 (1576) for param1, while the ALICE $2.76 \mathrm{TeV}$ measurement gives $1601 \pm 60$ [45]. The error band on our $5.44 \mathrm{TeV}$ prediction accounts for the normalization uncertainty as explained in the text. 


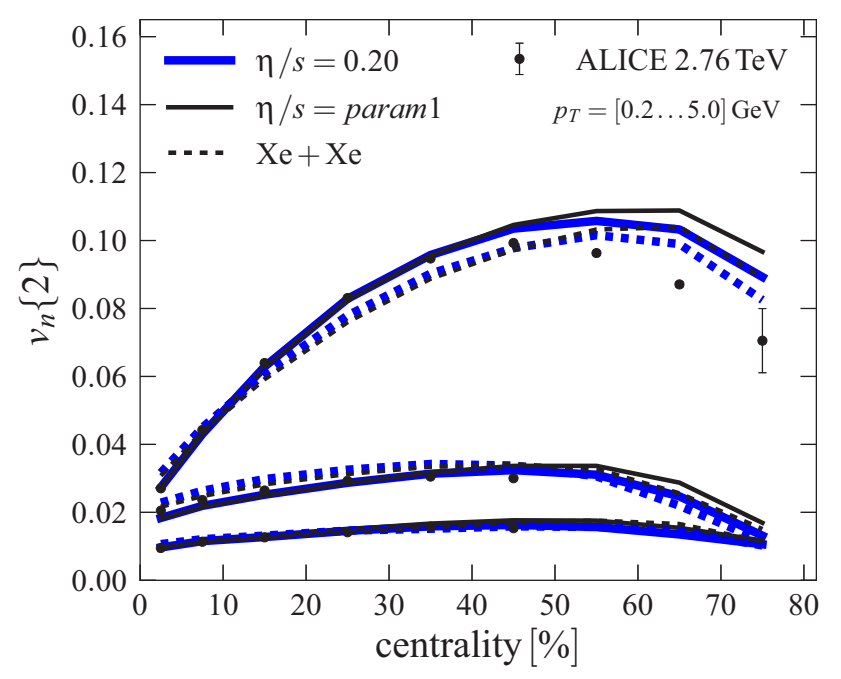

FIG. 4. The NLO EbyE EKRT model prediction for the centrality dependence of charged hadron flow harmonics $v_{n}\{2\}$ in $5.44 \mathrm{TeV}$ $\mathrm{Xe}+\mathrm{Xe}$ collisions at the LHC, computed for the $\eta / s(T)$ parametrizations of Fig. 1. The results from Ref. [18] and ALICE data [49] for $2.76 \mathrm{TeV} \mathrm{Pb}+\mathrm{Pb}$ collisions are also shown.

For the $\eta / s=0.2$ case the result is essentially the same. The error band on our $5.44 \mathrm{TeV}$ prediction in Fig. 3 is obtained with this procedure, applying the same relative normalization uncertainty for all centralities and individually for both $\eta / s$ parametrizations.

Our 5.44 $\mathrm{TeV} \mathrm{Xe}+\mathrm{Xe}$ prediction for the centrality dependence of the two-particle cumulant flow harmonics $v_{n}\{2\}$ of charged hadrons is shown in Fig. 4 along with our earlier results for $2.76 \mathrm{TeV} \mathrm{Pb}+\mathrm{Pb}$ collisions [18] and the corresponding ALICE data [49]. Perhaps not readily expected, the $v_{2}\{2\}$ in $\mathrm{Xe}+\mathrm{Xe}$ collisions is predicted to increase more slowly towards peripheral collisions than that in the $\mathrm{Pb}+\mathrm{Pb}$ system. We also predict that from central to semiperipheral collisions $v_{3}\{2\}$ is larger in $\mathrm{Xe}+\mathrm{Xe}$ than in $\mathrm{Pb}+\mathrm{Pb}$, which could be expected since the $v_{3}\{2\}$ originates from the initial density fluctuations of the

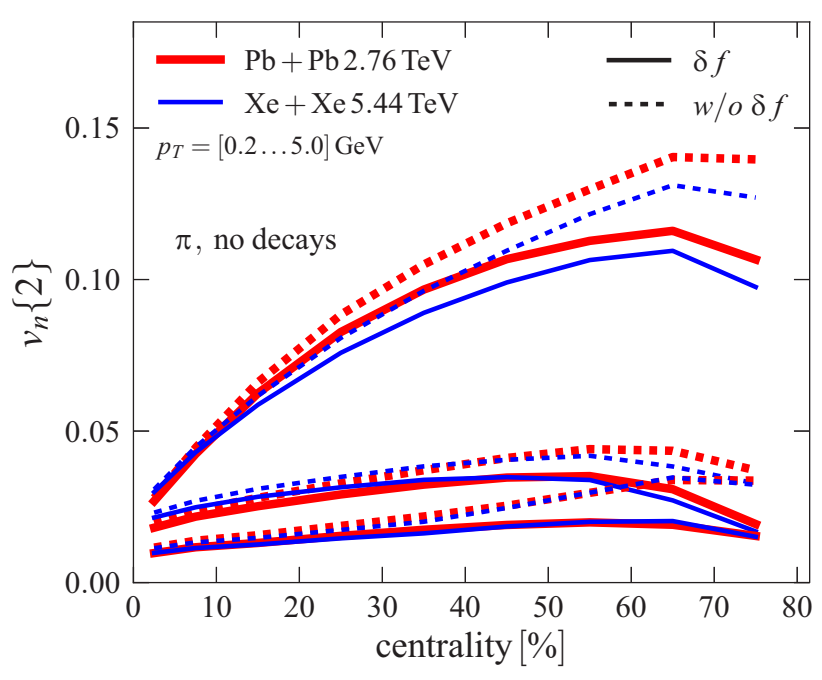

FIG. 6. The effect of the $\delta f$ corrections in the two-particle cumulant flow harmonics in $5.44 \mathrm{TeV} \mathrm{Xe}+\mathrm{Xe}$ (thin blue curves) and $2.76 \mathrm{TeV} \mathrm{Pb}+\mathrm{Pb}$ collisions (thick red curves) vs. centrality. Only thermal pions (no pions from decays) are considered in this figure.

system which are larger in a smaller system. Finally, we predict $v_{4}\{2\}$ to be of the same magnitude in both systems but show a flattening of the centrality slope in $\mathrm{Xe}+\mathrm{Xe}$. To quantify the systematics of the predicted flow coefficients as well as the sensitivity to $\eta / s(T)$ in more detail, we also plot the corresponding ratios $v_{n}\{2\}(\mathrm{Xe}+\mathrm{Xe}, 5.44 \mathrm{TeV}) / v_{n}\{2\}(\mathrm{Pb}+\mathrm{Pb}, 2.76 \mathrm{TeV})$ in Fig. 5. We can see from Figs. 4 and 5 that a simultaneous analysis of the flow harmonics in $\mathrm{Xe}+\mathrm{Xe}$ and $\mathrm{Pb}+\mathrm{Pb}$ collisions shows more sensitivity to $\eta / s(T)$ than the analysis of $\mathrm{Pb}+\mathrm{Pb}$ collisions alone.

To analyze the predicted $v_{n}\{2\}$ systematics further, we study in Fig. 6 the effects of the $\delta f$ corrections for the $\mathrm{Xe}+\mathrm{Xe}$ and $\mathrm{Pb}+\mathrm{Pb}$ systems. We see that these effects are similar in the relative magnitude and that they remain acceptably small until $\sim 50 \%$ centralities in both systems. Thus, we can confirm that the predicted slope change of $v_{n}\{2\}$ (and of $v_{2}\{2\}$ in

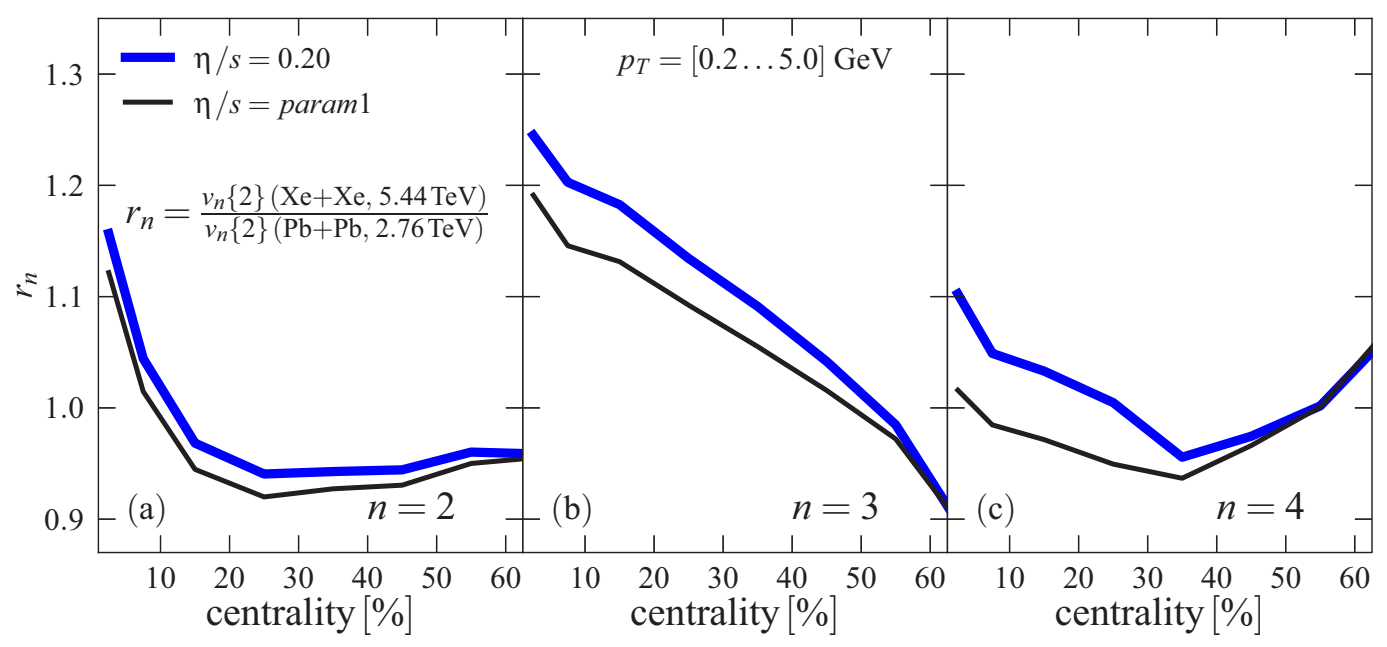

FIG. 5. Ratios of the predicted flow harmonics $v_{n}\{2\}$ in $5.44 \mathrm{TeV} \mathrm{Xe}+\mathrm{Xe}$ and $2.76 \mathrm{~Pb}+\mathrm{Pb}$ collisions, for each of the $\eta / s$ parametrizations studied. 

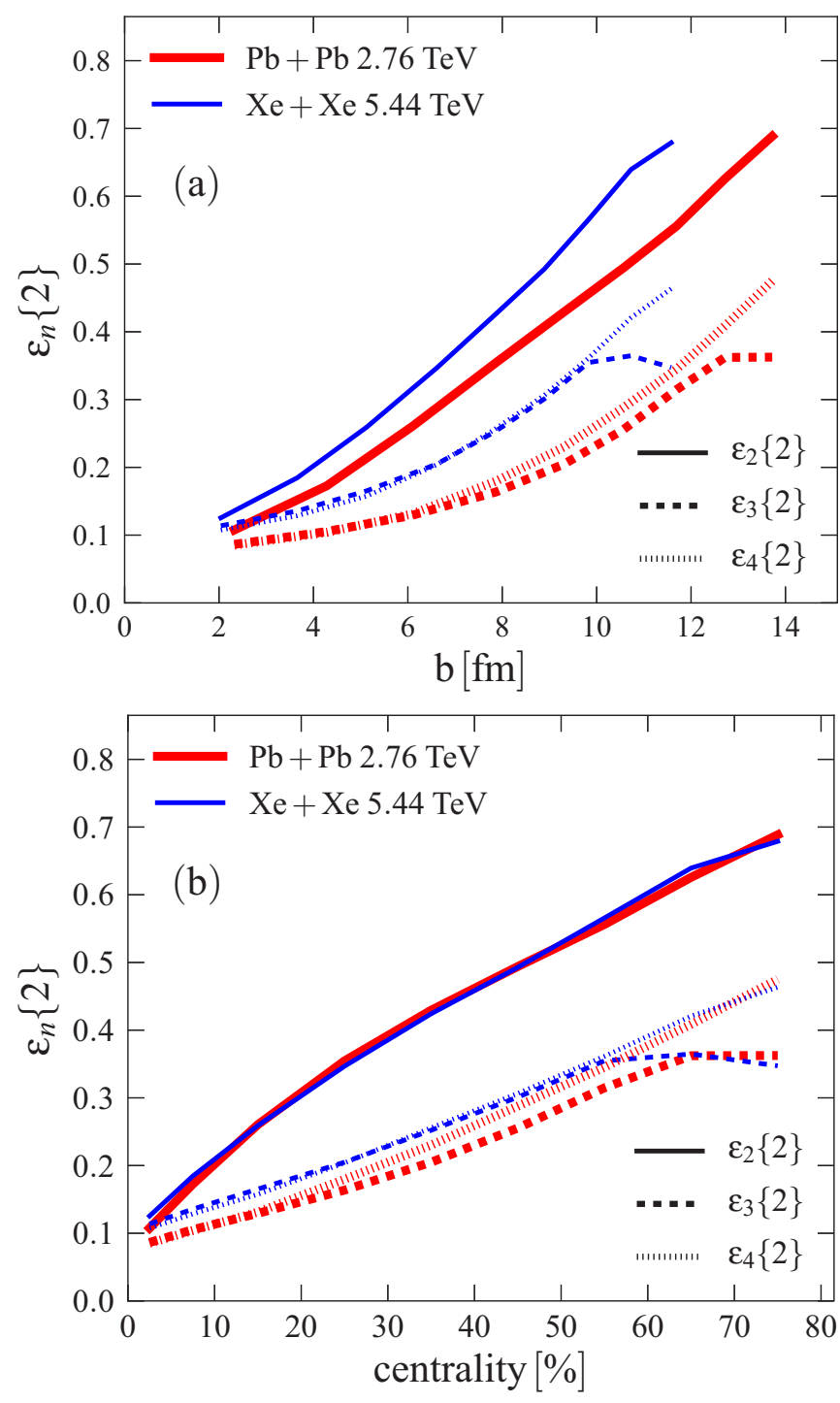

FIG. 7. Initial eccentricities $\varepsilon_{n}\{2\}$ in $5.44 \mathrm{TeV} \mathrm{Xe}+\mathrm{Xe}$ (thin blue curves) and $2.76 \mathrm{TeV} \mathrm{Pb}+\mathrm{Pb}$ collisions (thick red curves) vs. impact parameter (upper panel) and vs. centrality (lower panel).

particular) from $\mathrm{Pb}+\mathrm{Pb}$ to $\mathrm{Xe}+\mathrm{Xe}$ is indeed not caused by the $\delta f$ corrections.

Figure 7 then shows the initial eccentricities $\varepsilon_{n}\{2\}$ as a function of the impact parameter and centrality. As expected, $\varepsilon_{n}\{2\}$ against $|\mathbf{b}|$ shows larger eccentricities for the smaller system. However, when $\varepsilon_{n}\{2\}$ is considered against centrality, this systematics changes. This is because the average impact parameter in each centrality class is smaller for a smaller system. This relative shift in $\langle|\mathbf{b}|\rangle$ when moving from $\mathrm{Pb}+\mathrm{Pb}$ to $\mathrm{Xe}+\mathrm{Xe}$ collisions, together with the $|\mathbf{b}|$-slope systematics of $\varepsilon_{n}\{2\}$, explains why $\varepsilon_{2}\{2\}$ vs. centrality becomes of the same magnitude in both systems while $\varepsilon_{3,4}\{2\}$ remain slightly larger in the $\mathrm{Xe}+\mathrm{Xe}$ system. The fluid-dynamical evolution is then responsible for the rest, converting the initial $\varepsilon_{n}\{2\}$ into the final-state $v_{n}\{2\}$ the more efficiently the longer the system evolves in the QGP phase where the pressure gradients are large. Thus, towards peripheral $\mathrm{Xe}+\mathrm{Xe}$ collisions, and with larger $\eta / s$ in the QGP, the $\varepsilon_{n}\{2\} \rightarrow v_{n}\{2\}$ conversion efficiency decreases. This explains why the $v_{n}\{2\}$ slopes from central towards peripheral collisions are smaller in the $\mathrm{Xe}+\mathrm{Xe}$ system than in the $\mathrm{Pb}+\mathrm{Pb}$ system, and why the $v_{n}\{2\}$ for $\eta / s=0.2$ are larger than those for param1.

\section{CONCLUSIONS}

We have computed the centrality dependencies of the charged hadron multiplicity and two-particle cumulant flow harmonics in $5.44 \mathrm{TeV} \mathrm{Xe+Xe} \mathrm{collisions} \mathrm{at} \mathrm{the} \mathrm{LHC} \mathrm{by}$ applying the machinery and predictive power of the NLO EbyE EKRT model, and using our two best-fitting parametrizations of $\eta / s(T)$ of Ref. [18]. The corresponding multiplicity now (October 2017) measured at the LHC will serve as a most welcome first constraint for the $A$ systematics of the primary QGP production mechanism at highest collision energies so far. The measurement of flow harmonics in $\mathrm{Xe}+\mathrm{Xe}$ collisions at $5.44 \mathrm{TeV}$ will in turn offer further constraints for the space-time evolution and $\eta / s(T)$ of QCD matter. The predicted centrality dependence of the flow harmonics in $\mathrm{Xe}+\mathrm{Xe}$ collisions shows interesting systematics: The centrality slopes of $v_{n}\{2\}$ are in general smaller in $\mathrm{Xe}+\mathrm{Xe}$ than in $\mathrm{Pb}+\mathrm{Pb}$. From $10 \%$ central collisions onwards the predicted $v_{2}\{2\}$ in $\mathrm{Xe}+\mathrm{Xe}$ becomes smaller than in $\mathrm{Pb}+\mathrm{Pb}$ collisions, while $v_{3}\{2\}$ remains larger than in $\mathrm{Pb}+\mathrm{Pb}$ until $50 \%$ centralities and $v_{4}\{2\}$ is of the same magnitude in both systems. We look forward to seeing whether the measurements confirm these predictions, especially the $\mathrm{Pb}+\mathrm{Pb} \rightarrow \mathrm{Xe}+\mathrm{Xe}$ systematics of the flow harmonics, which we believe is due to a decreasing efficiency in converting the initial spatial asymmetries (eccentricities) into the final state momentum asymmetries (flow coefficients).

\section{ACKNOWLEDGMENTS}

K.J.E. and K.T. are supported by the Academy of Finland, Projects No. 297058 and No. 310130, correspondingly. H.N. is supported by the EUs Horizon 2020 research and innovation programme under the Marie Sklodowska-Curie grant agreement No. 655285, and by the Collaborative Research Center CRC-TR 211 Strong-interaction matter under extreme conditions funded by DFG. R.P. is supported by the European Research Council, grant No. 725369. We acknowledge the CSC-IT Center for Science in Espoo, Finland, for the allocation of the computational resources. We also thank the members of the ALICE collaboration who drew our attention to the new $\mathrm{Xe}+\mathrm{Xe}$ run, which motivated this study.
[1] K. J. Eskola, K. Kajantie, and J. Lindfors, Nucl. Phys. B 323, 37 (1989).
[2] L. D. McLerran and R. Venugopalan, Phys. Rev. D 49, 2233 (1994). 
[3] K. J. Eskola, K. Kajantie, P. V. Ruuskanen, and K. Tuominen, Nucl. Phys. B 570, 379 (2000).

[4] D. Kharzeev and M. Nardi, Phys. Lett. B 507, 121 (2001).

[5] D. Kharzeev and E. Levin, Phys. Lett. B 523, 79 (2001).

[6] T. Lappi, Phys. Rev. C 67, 054903 (2003).

[7] H.-J. Drescher and Y. Nara, Phys. Rev. C 75, 034905 (2007).

[8] F. Gelis, E. Iancu, J. Jalilian-Marian, and R. Venugopalan, Annu. Rev. Nucl. Part. Sci. 60, 463 (2010).

[9] J. L. Albacete and A. Dumitru, arXiv:1011.5161 [hep-ph].

[10] R. Paatelainen, K. J. Eskola, H. Holopainen, and K. Tuominen, Phys. Rev. C 87, 044904 (2013).

[11] R. Paatelainen, K. J. Eskola, H. Niemi, and K. Tuominen, Phys. Lett. B 731, 126 (2014).

[12] B. Schenke, S. Jeon, and C. Gale, Phys. Rev. Lett. 106, 042301 (2011).

[13] C. Gale, S. Jeon, B. Schenke, P. Tribedy, and R. Venugopalan, Phys. Rev. Lett. 110, 012302 (2013).

[14] B. Schenke, P. Tribedy, and R. Venugopalan, Phys. Rev. Lett. 108, 252301 (2012).

[15] T. Pierog, I. Karpenko, J. M. Katzy, E. Yatsenko, and K. Werner, Phys. Rev. C 92, 034906 (2015).

[16] C. Shen, Z. Qiu, H. Song, J. Bernhard, S. Bass, and U. Heinz, Comput. Phys. Commun. 199, 61 (2016).

[17] I. A. Karpenko, P. Huovinen, H. Petersen, and M. Bleicher, Phys. Rev. C 91, 064901 (2015).

[18] H. Niemi, K. J. Eskola, and R. Paatelainen, Phys. Rev. C 93, 024907 (2016).

[19] S. Ryu, J.-F. Paquet, C. Shen, G. S. Denicol, B. Schenke, S. Jeon, and C. Gale, Phys. Rev. Lett. 115, 132301 (2015).

[20] J. Noronha-Hostler, M. Luzum, and J. Y. Ollitrault, Phys. Rev. C 93, 034912 (2016).

[21] G. Giacalone, L. Yan, J. Noronha-Hostler, and J. Y. Ollitrault, Phys. Rev. C 94, 014906 (2016).

[22] F. G. Gardim, F. Grassi, M. Luzum, and J. Noronha-Hostler, Phys. Rev. C 95, 034901 (2017).

[23] G. Giacalone, J. Noronha-Hostler, M. Luzum, and J.-Y. Ollitrault, Phys. Rev. C 97, 034904 (2018).

[24] J. Novak, K. Novak, S. Pratt, J. Vredevoogd, C. E. ColemanSmith, and R. L. Wolpert, Phys. Rev. C 89, 034917 (2014).

[25] S. Pratt, E. Sangaline, P. Sorensen, and H. Wang, Phys. Rev. Lett. 114, 202301 (2015).

[26] J. E. Bernhard, J. S. Moreland, S. A. Bass, J. Liu, and U. Heinz, Phys. Rev. C 94, 024907 (2016).
[27] S. A. Bass, J. E. Bernhard, and J. S. Moreland, Nucl. Phys. A 967, 67 (2017).

[28] J. E. Bernhard, J. S. Moreland, and S. A. Bass, Nucl. Phys. A 967, 293 (2017).

[29] J. Auvinen, I. Karpenko, J. E. Bernhard, and S. A. Bass, arXiv:1706.03666 [hep-ph].

[30] H. Niemi, K. J. Eskola, R. Paatelainen, and K. Tuominen, Phys. Rev. C 93, 014912 (2016).

[31] K. J. Eskola, H. Niemi, R. Paatelainen, and K. Tuominen, Nucl. Phys. A 967, 313 (2017).

[32] Z. Kunszt and D. E. Soper, Phys. Rev. D 46, 192 (1992).

[33] K. J. Eskola and K. Tuominen, Phys. Lett. B 489, 329 (2000).

[34] K. J. Eskola and K. Tuominen, Phys. Rev. D 63, 114006 (2001).

[35] R. K. Ellis and J. C. Sexton, Nucl. Phys. B 269, 445 (1986).

[36] R. Paatelainen, Ph.D. thesis, Research Report No. 8/2014, University of Jyäskylä, arXiv:1409.3508 [hep-ph].

[37] J. Pumplin, D. R. Stump, J. Huston, H.-L. Lai, P. Nadolsky, and W.-K. Tung, J. High Energy Phys. 07 (2002) 012.

[38] I. Helenius, K. J. Eskola, H. Honkanen, and C. A. Salgado, J. High Energy Phys. 07 (2012) 073.

[39] K. J. Eskola, K. Kajantie, and K. Tuominen, Nucl. Phys. A 700, 509 (2002).

[40] J. R. Cudell, V. V. Ezhela, P. Gauron, K. Kang, Y. V. Kuyanov, S. B. Lugovsky, E. Martynov, B. Nicolescu, E. A. Razuvaev, and N. P. Tkachenko (COMPETE Collaboration), Phys. Rev. Lett. 89, 201801 (2002).

[41] G. Antchev et al. (TOTEM Collaboration), Europhys. Lett. 101, 21004 (2013).

[42] G. S. Denicol, H. Niemi, E. Molnar, and D. H. Rischke, Phys. Rev. D 85, 114047 (2012); 91, 039902(E) (2015).

[43] E. Molnar, H. Niemi, G. S. Denicol, and D. H. Rischke, Phys. Rev. D 89, 074010 (2014).

[44] P. Huovinen and P. Petreczky, Nucl. Phys. A 837, 26 (2010).

[45] K. Aamodt et al. (ALICE Collaboration), Phys. Rev. Lett. 106, 032301 (2011).

[46] J. Adam et al. (ALICE Collaboration), Phys. Rev. Lett. 116, 222302 (2016).

[47] B. I. Abelev et al. (STAR Collaboration), Phys. Rev. C 79, 034909 (2009).

[48] S. S. Adler et al. (PHENIX Collaboration), Phys. Rev. C 71, 034908 (2005); 71, 049901(E) (2005).

[49] K. Aamodt et al. (ALICE Collaboration), Phys. Rev. Lett. 107, 032301 (2011). 